\title{
LES ORAGES
}

\section{Météorologie des grains, de la grêle et des éclairs}

\author{
Frank Roux
}

Un livre sur la foudre (voir la description du livre de Gary ${ }^{(1)}$ ), un autre sur les orages : à première vue, les contenus doivent être proches et il est bien tentant de décrire celui-ci par rapport à celui-là. Mais, si compétition il y a entre les deux ouvrages, celui de Frank Roux ne part pas gagnant : plus ancien (1991), pas de papier glacé, pas de couleurs, pas de photos, juste quelques

Documents Payot

\section{Frank Roux \\ Les orages}

Météorologie des grains, de la grêle et des éclairs

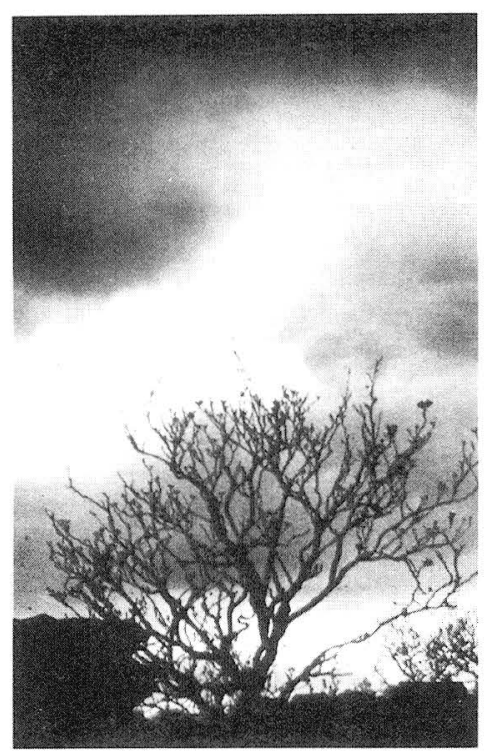

schémas au trait pour illustrer.

Le contenu aussi est plus austère : pas de première partie historique, pas d'anecdotes, pas d'expériences décrites en détail. De plus, l'inclusion dans le texte lui-même de nombreuses références bibliographiques fait de cette publication un ouvrage destiné plutôt aux professionnels.

Mais on aurait tort d'en rester là. Les orages est un livre différent et tout à fait complémentaire du précédent. Le cumulonimbus, nuage d'orage par excellence, y est examiné sous tous ses aspects, de l'échelle synoptique à l'échelle microscopique.

Une première partie décrit en détail le « décor »: description de l'atmosphère, composition de l'air, circulation générale, phénomènes de grande échelle et effets locaux. Puis, l'orage est replacé dans son contexte : situations météorologiques favorables, description détaillée de la répartition dans le monde et sur le territoire français. Cette première partie comprend également un chapitre entier sur les moyens d'observation et d'étude, chapitre complété par une annexe en fin d'ouvrage sur les mesures en météorologie.

La deuxième partie s'attache à décrire la formation des cumulonimbus et les mouvements à l'intérieur des nuages de ce type. Pilotes d'avion, passionnés d'ULM, de deltaplane et de parapente trouveront là une foule de choses intéressantes pour mieux comprendre l'aérologie. L'auteur, s'il va parfois loin dans les explications théoriques, sait aussi rester pratique et n'oublie pas de donner des indications visuelles pour tenter de classer le nuage orageux dans telle ou telle catégorie.

La dernière partie s'intitule « Bruits et fureurs », tout un programme ! On y apprend tout ce qu'il est possible sur les tornades. Le chapitre sur la grêle présente les différentes théories soutenues par les uns ou les autres et montre bien que c'est un sujet sur lequel on ignore encore à peu près tout. Le chapitre sur les éclairs, qui termine l'ouvrage, est en fait la seule partie commune avec le livre de Gary, et l'on y trouve, en condensé, l'essentiel de ce dernier.

(1) La Météorologie a publié une note de lecture sur La foudre, de C. Gary, dans le $\mathrm{n}^{\circ} 10$ de juin 1995 (p. 85). 
Docteur ès sciences et directeur de recherches au CNRS, l'auteur travaille au Laboratoire d'aérologie du CNRS à Toulouse (et au comité de rédaction de $L a$ Météorologie, Ndlr). Avec Les orages, il a réalisé un travail d'une grande objectivité présentant à chaque fois les différentes théories en cours lorsque l'unanimité des scientifiques n'a pas encore pu se faire.

Frank Roux signe là un excellent ouvrage, très complet, où tous les aspects de l'orage sont examinés en détail.

Michel Hontarrède

Les orages. Météorologie des grains, de la grêle et des éclairs.

Par Frank Roux.

Payot, Paris, 1991,358 p. 140 F.

Ndlr : Cette note de lecture est parue dans Met Mar, 1995, 166, p. 48. Nous remercions la rédaction de Met Mar de nous avoir autorisés à la reproduire ici. 